\title{
Greener Synthesis, Characterization, and Antimicrobiological Effects of Helba Silver Nanoparticle-PMMA Nanocomposite
}

\author{
Manal A. Awad (D), ${ }^{1}$ Awatif A. Hendi $\left(\mathrm{D},{ }^{2}\right.$ Khalid M. O. Ortashi $\left(\mathrm{D},{ }^{3}\right.$ Amnah B. Alanazi (D), \\ Batool A. ALZahrani, ${ }^{2}$ and Dina A. Soliman ${ }^{4}{ }^{4}$ \\ ${ }^{1}$ King Abdullah Institute for Nanotechnology, King Saud University, Riyadh 11451, Saudi Arabia \\ ${ }^{2}$ Department of Physics, King Saud University, Riyadh 11495, Saudi Arabia \\ ${ }^{3}$ Department of Chemical Engineering, King Saud University, Riyadh 11421, Saudi Arabia \\ ${ }^{4}$ Department of Microbiology, Faculty of Science, King Saud University, Riyadh 11459, Saudi Arabia
}

Correspondence should be addressed to Manal A. Awad; a.manalawad@gmail.com and Awatif A. Hendi; ahindi@ksu.edu.sa

Received 3 October 2018; Revised 3 December 2018; Accepted 12 December 2018; Published 21 April 2019

Academic Editor: Cornelia Vasile

Copyright @ 2019 Manal A. Awad et al. This is an open access article distributed under the Creative Commons Attribution License, which permits unrestricted use, distribution, and reproduction in any medium, provided the original work is properly cited.

\begin{abstract}
Nanocomposites are characterized as a multiphase material where one of the phases has a dimension in the nanoscale. There has been huge enthusiasm for the commercialization of nanocomposites for an assortment of uses including medicinal, electronic, and basic. The general motivation behind this study was on the development of silver nanoparticles, due to the present enthusiasm encompassing these metals due to their exceptional properties which are not quite the same as the relating bulk material. A novel, simple, cost-effective, nontoxic, and environmentally friendly technique was developed for synthesizing silver nanoparticle- (AgNP-) poly(methyl methacrylate) (PMMA) nanocomposite using Trigonella foenum-graecum (Helba) aqueous extract. UV-visible spectroscopic analysis was carried out to assess the formulation of AgNPs. The particle size distribution of AgNPs was determined by dynamic light scattering (DLS). The average size of green AgNPs was about $83 \mathrm{~nm}$. Images of spherical green nanoparticles were characterized using transmission electron microscopy (TEM). The resultant green AgNPs were added slowly to polymer (PMMA) solution. The AgNPs encapsulated within the polymer chains were characterized by X-ray diffraction (XRD) and Fourier transform infrared spectroscopy (FTIR). Modification of thermal stabilities of AgNP/PMMA nanocomposites was confirmed using thermogravimetric analysis (TGA). The green AgNP/PMMA nanocomposites showed improved thermal stabilities. The green AgNP/PMMA nanocomposite film proved antimicrobial in water microbiological testing. Thus, the key findings of the work include the use of a safe and simple nanocomposite, which had marked antibacterial activity and potential application in water filtration.
\end{abstract}

\section{Introduction}

Nanomaterials are the most studied materials of the century that gave birth to a new branch of science known as "nanotechnology." Nanomaterials are prepared from bulk size materials, but the smaller size and shape of these particles differentiate their chemical actions from those of their parent material [1]. The smaller size of nanomaterials helps them to penetrate specific cellular locations, and their additional surface area facilitates increased adsorption and targeted delivery of substances [2]. Nanomaterials exist in volcanic dust, mineral composites, and anthropogenic waste materials like coal combustion, diesel exhaust, and welding fumes (incidental nanomaterials) [3]. Engineered nanomaterials manufactured with nanoscale dimension are generally grouped into four types: carbon, metals, metal oxides, dendrimers, and composites [4].

Silver nanoparticles (AgNPs) have unique optical, electri$\mathrm{cal}$, and thermal properties and are incorporated into products that range from photovoltaics to biological and chemical sensors. Some examples include conductive inks, pastes, and fillers, which utilize AgNPs for their high electrical conductivity, stability, and low sintering temperature. Additional applications include molecular diagnostics and photonic devices, which take advantage of the novel optical properties of these nanomaterials. An increasingly common 
application is the use of AgNPs for antimicrobial coatings; textile products, keyboards, wound dressings, and biomedical devices now contain AgNPs that continuously release a low level of silver ions to provide protection against bacteria [5].

Polymers are host materials for metal nanoparticles [6]. The polymer acts as surface topping specialist when nanoparticles are implanted in them. The nanocomposites obtained display upgraded optical properties [7]. However, the properties of polymer composites depend on the type of nanoparticles incorporated and their size, shape, concentration, and interaction with the polymer matrix. Poly(methyl methacrylate) (PMMA) is a polymeric glass with a wide range of applications. The use of PMMA offers twofold advantages such as availability of carboxylate functional groups for chemical bonding with metal ions and high solubility of PMMA in solvents like DMF for silver nitrate reduction [8].

Owing to their diverse properties of polymer nanocomposites such as unprecedented performance, improved properties compared to the constituent parts, design flexibility, and lower life cycle costs, they have attracted considerable attention. The nanocomposite makes use of organic/inorganic nanoparticles incorporated in polymers that produce new materials with a potential application in catalysis, bioengineering, photonics, electronics, and antibacterial activities [9-11]. AgNPs have been shown to form composites with polymers, such as polyvinyl alcohol, polypyrrole, polyvinylidene fluoride, chitosan, and cellulose. For a silver-polymer nanocomposite, it is important to maintain a controllable size of the nanoparticles in the matrix along with a uniform distribution of the same in the polymer matrix. The synthesis of the silver-polymer nanocomposites includes the mixing of a nanoparticle solution with the polymerization mixture. These silver-polymer nanocomposites can have a wide range of applications such as in biomedicine, textiles, water treatment, food storage containers, home appliances, and medical devices $[12,13]$.

There are various modes of synthesis of nanomaterials which include chemical, physical, and biological. Some chemical and physical methods which have been employed have contributed to environmental contamination, since the chemical procedures involved can generate a large amount of hazardous byproducts. Thus, there is a need to develop new "green" synthesis procedures for nanoparticles which are ecofriendly, safe, and nontoxic procedures to be carried out at low energy and temperature. The biological methods include synthesis of nanomaterials from the extracts of plants, bacteria, and fungal species, among other procedures $[14,15]$.

In this study, stable AgNPs were synthesized by the reduction of silver nitrate with Trigonella foenum-graecum aqueous extract. The AgNPs were characterized by transmission electron microscopy (TEM), UV-Vis spectra, and zetasizer. The AgNP/PMMA nanocomposite was characterized via FTIR and XRD, and its thermal stability was assessed using TGA. In addition, the antimicrobial effect of PMMA nanocomposites was evaluated on tap water. The main objective of the study was to use the AgNPs in PMMA nanocomposites as a biofilter for tap water.

\section{Materials and Methods}

2.1. Greener Synthesis of AgNPs. Trigonella foenum-graecum (Helba, $3 \mathrm{~g}$ ) seeds were purchased from the local market in Riyadh City (Saudi Arabia). The seeds were washed several times to remove the dust on the periphery of the seed and then were dried and soaked in $90 \mathrm{~mL}$ boiled distilled water overnight. The extract was passed through Whatmann no. 1 filter paper, and the combined filtrate was immediately used for the preparation of nanoparticles. The resultant aqueous filtrate was treated with aqueous solution of silver nitrate $\left(\mathrm{AgNO}_{3}\right)$.

Silver nitrate $(1 \mathrm{mmol} / \mathrm{mL}$, analytical grade, Techno Pharmchem, India) was dissolved in $50 \mathrm{~mL}$ distilled water with vigorous stirring at $80^{\circ} \mathrm{C}$ for $5 \mathrm{~min}$. Then, $5 \mathrm{~mL}$ of $\mathrm{Helba}$ extract was added to the silver nitrate solution. The colloidal solution changed in color within an hour, which confirmed the reduction of $\mathrm{Ag}$ ions and the formation of greener AgNPs. The change in color of the reaction was noted by visual observation. Then, the green nanoparticle solution was incubated at room temperature until it was used.

2.2. Preparation of Green AgNP/PMMA Nanocomposite Film. PMMA (6g, SABIC Company, Saudi Arabia) was dissolved in $50 \mathrm{~mL}$ of N,N-dimethylformamide (DMF, R\&M Marketing, Essex, UK) with continuous stirring for 3 hours at $80 \mathrm{C}$. After that, $3 \mathrm{~mL}$ of freshly prepared solution of green AgNPs (previous section) was added with constant stirring at $80^{\circ} \mathrm{C}$ and $8000 \mathrm{rpm}$. This mixture was further stirred for $1 \mathrm{~h}$ to complete the reaction. The whole previous processes were carried out in a fume hood. A light brown solution was obtained due to the formation of silver colloid. At that point, the solution was cast on a glass plate and DMF was allowed to evaporate at room temperature to create a nanocomposite film. The film was air dried under a fume hood. The film was washed with methanol to remove residual DMF and to promote drying and was removed from the glass plate after drying.

2.3. Characterization of Green Nanoparticles and Nanocomposite. X-ray diffraction (XRD) (Bruker D8 Discover) scanning was performed to identify the green AgNP/PMMA nanocomposite and PMMA films. TGA analyses of green AgNP/PMMA nanocomposite and PMMA films were carried out in a thermal system (Mettler Toledo TGA/DSC 1). About $4 \mathrm{mg}$ dried film was used for the TGA experiment. TGA thermograms were obtained in the range $0-800^{\circ} \mathrm{C}$ under nitrogen flow at a rate of $10^{\circ} \mathrm{C} \mathrm{min}^{-1}$. Their distinct graphs were plotted with weight (percentage) loss and heat flow against temperature.

The size of the synthesized green AgNPs was analyzed through a zetasizer (Nano series, HT Laser, ZEN3600 Molvern Instrument, UK). Transmission electron microscopy (JEM-1011, JEOL, Japan) was employed to characterize the size, shape, and morphology of the formed green synthesized nanoparticles at an accelerating voltage of $100 \mathrm{kV}$.

\subsection{Water Microbiological Testing}

2.4.1. Plate Count Method. The microbial growth in the treated tap water was assessed using the plate count method. 
The plate count method relies on bacteria growing a colony on a nutrient medium so that the colony becomes visible to the naked eye and the number of colonies on a plate can be counted. To be effective, the dilution of the original sample must be arranged so that on average colonies of the target bacterium between 30 and 300 are grown. To ensure that an appropriate number of colonies will be generated, several dilutions are normally cultured. This approach is widely utilized for the evaluation of the effectiveness of water treatment by the inactivation of representative microbial contaminants such as E. coli following $[16,17]$.

To treat tap water with the green nanocomposite film, a $1 \times 1 \mathrm{~cm}$ green film was soaked in $50 \mathrm{~mL}$ tap water in an Erlenmeyer flask and kept for $48 \mathrm{~h}$.

Three different types of media, eosin methylene blue (E.M.B) agar, nutrient agar (N.A) for Gram-negative bacteria such as E. coli, and Muller-Hinton (M.H) agar, have been used to obtain many isolated microorganisms. The water samples evaluated were normal tap water as control (NW) and treated tap water (TW).

To prepare $250 \mathrm{~g}$ of nutrient agar media, $7 \mathrm{~g}$ of the agar media was dissolved in $250 \mathrm{~mL}$ of distilled water and autoclaved. To prepare $250 \mathrm{~g}$ from MacConkey agar, $12.87 \mathrm{~g}$ of the agar medium was dissolved in $250 \mathrm{~mL}$ of distilled water and then autoclaved. For Muller-Hinton agar media, $9.5 \mathrm{~g}$ of the media was dissolved in $250 \mathrm{~mL}$ of distilled water and then autoclave it. After this, $1 \mathrm{~mL}$ from both treated and untreated tap water (TW or NW) was added to the assigned petri dishes. Thereafter, the media were thoroughly mixed, autoclaved, and incubated upside down at $37^{\circ} \mathrm{C}$ for $24-48$ hours.

\section{Results and Discussion}

3.1. Visual Observation and UV-Vis Spectroscopy Analysis. Noble metals display exceptional optical properties because of surface plasmon resonance (SPR) [18]. The development of AgNPs was first checked for change in color from colorless to brown and UV-Vis spectroscopy. The change in color showed the formation of AgNPs because of the reduction of silver metal particles $\mathrm{Ag}^{+}$to nanoparticles $\mathrm{Ag}^{0}$ [19]. This color refers to the excitation of SPR. As shown in Figure 1, a characteristic SPR band for AgNPs was observed at around $339 \mathrm{~nm}$.

3.2. TEM and Particle Size Analysis. TEM images presented monodisperse AgNPs with spherical shape, as shown in Figure 2(a). The average particle size was determined by dynamic light scattering (DLS) and was found to be $83.01 \mathrm{~nm}$ as revealed, in the size distribution graph that showed monodisperse AgNPs (Figure 2(b)). These results agreed with and confirmed the results obtained by TEM. These results are in agreement with Goyal et al. who teach that the size determination studies using DLS revealed the synthesized silver nanoparticles using Trigonella foenumgraecum seed extract size between 95 and $110 \mathrm{~nm}$ [20].

3.3. X-Ray Diffraction Analysis. The structures of pure PMMA and AgNPs in the polymer matrix were investigated

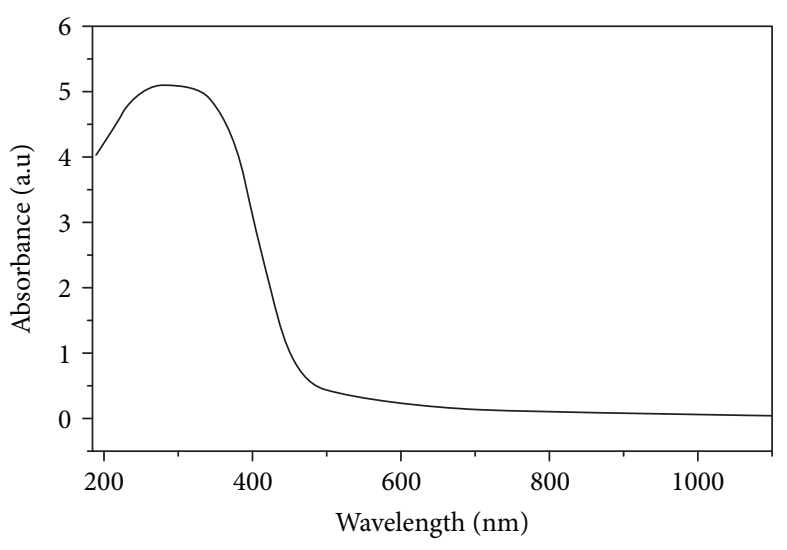

FIGURE 1: UV-visible absorption spectrum of silver nanoparticles prepared using a greener synthesis process.

using XRD analysis. Therefore, the XRD patterns of pure PMMA polymer and greener AgNPs/polymer nanocomposites were obtained as shown in Figures 3(a) and 3(b).

From Figure 3(a), it seems clear that pure PMMA thin film possessed no crystalline structure. Therefore, we can say that it had an amorphous structure. As observed in Figure 3(b), the greener AgNP/PMMA nanocomposites exhibited and confirmed the existence of silver in the polymer matrix. It shows that in all the peaks, the metallic pure silver had spherical structure. Refraction was provided by many peaks at about $2 \theta=35-70^{\circ}$ while the first and second peaks were assigned to the lattice planes $\left(\begin{array}{lll}1 & 1 & 1\end{array}\right)$ and $\left(\begin{array}{lll}2 & 0 & 0\end{array}\right)$, respectively [21-23]. In addition to that, the polymer/AgNP composite patterns exhibited a two-phase (crystalline and amorphous) structure. The polymer/AgNP composite exhibited broad reflection and typical amorphous nature of the polymer, as expected, and the typical pattern of the face-centered cubic (FCC) Ag crystalline structure indicated the formation of metallic Ag [24].

The XRD pattern of PMMA showed that three broad peaks appeared, which corresponded to a mixture of ordered and disordered structures of the amorphous phase of the polymer [25]. The amorphous halo was caused by the spacing of individual polymer chains. Comparison of the diffraction patterns of PMMA and greener AgNP/PMMA nanocomposite showed that the peaks corresponding to PMMA became broader and seemed to disappear because of small AgNPs embedded in the PMMA chains [26].

3.4. FTIR Spectroscopy Analysis. To verify that the resultant composites contained PMMA, we determined the FTIR spectra of pure PMMA and the AgNP/PMMA composite, respectively. The results are shown in Figures 4(a) and 4(b). As can be seen in Figure 4(a), the bands between 1550 and $1800 \mathrm{~cm}^{-1}$ in pure PMMA were due to acrylate carboxyl groups or $\mathrm{C}=\mathrm{O}$ vibrations, which are all typical bands of PMMA. The peak at $3000-3007 \mathrm{~cm}^{-1}$ was assigned to the $\mathrm{C}-\mathrm{H}$ stretching vibration. For the greener AgNP/PMMA nanocomposite, the appearance of the peak was assigned to $\mathrm{C}=\mathrm{O}$ vibrations at $1550-1800 \mathrm{~cm}^{-1}$ (Figure $4(\mathrm{~b})$ ). From the spectra of Figure 4(a), it can be seen that the band at 


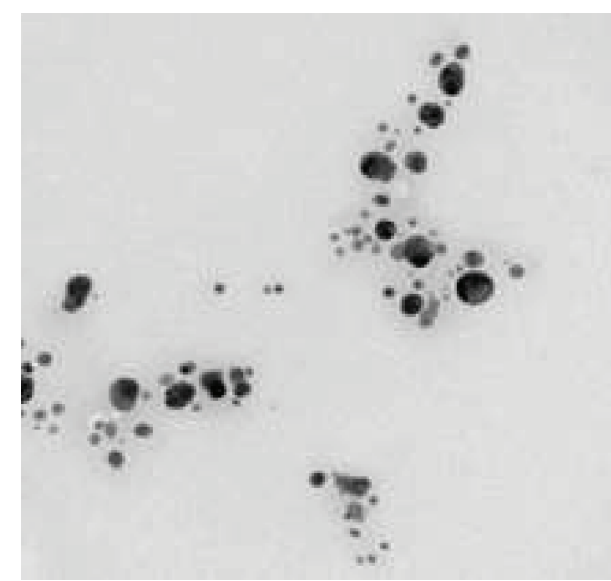

(a)

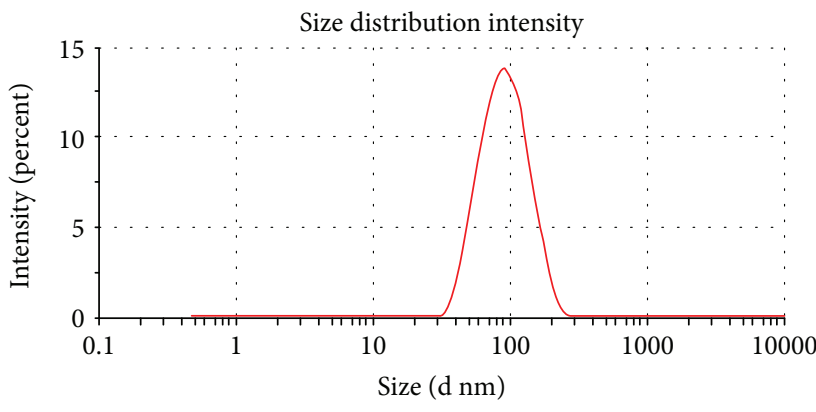

(b)

FIGURE 2: (a) TEM images of greener silver nanoparticles; the scale bar corresponds to $100 \mathrm{~nm}$. (b) Average particle size distribution curve determined by DLS.

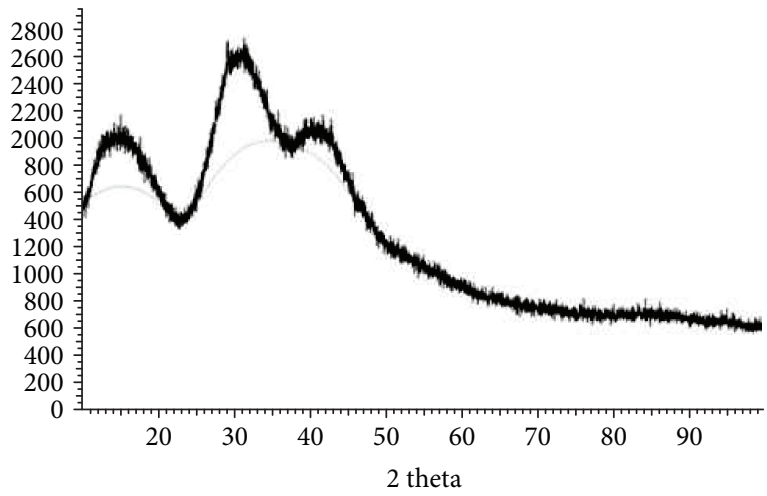

(a)

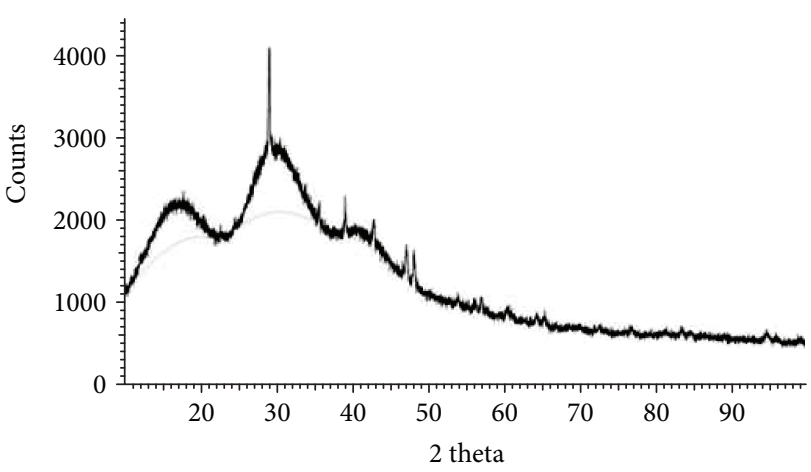

(b)

FIgURE 3: XRD patterns of (a) pure PMMA and (b) greener AgNP/PMMA nanocomposite.

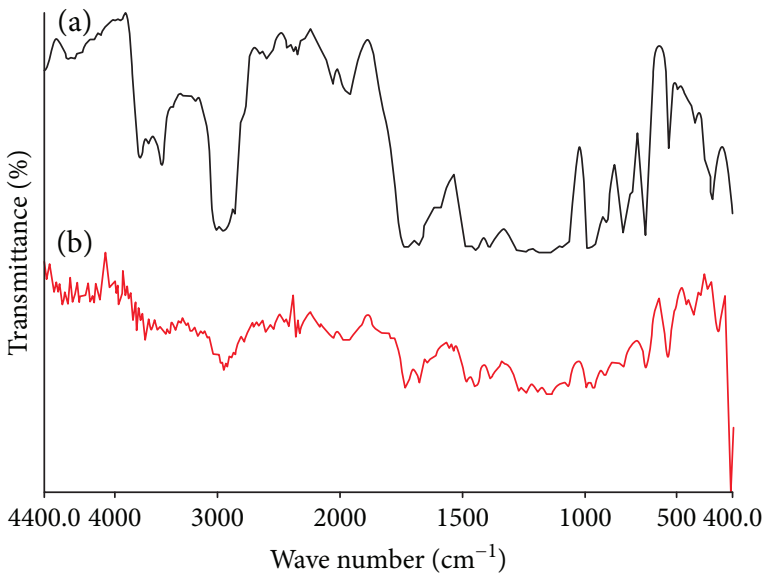

FIgURE 4: FTIR spectra of (a) pure PMMA and (b) Ag/PMMA nanocomposite.

$1,203 \mathrm{~cm}^{-1}$ originated from the $\mathrm{C}-\mathrm{O}-\mathrm{C}$ group. However, compared to Figure 4(a) for pure PMMA, the peak positions of Figure 4(b) for greener AgNP/PMMA shifted because of strong interaction between PMMA and AgNPs. For the AgNP/PMMA nanocomposite (Figure 4(b)), the peaks at $550-650 \mathrm{~cm}^{-1}$ were from the Ag-C stretching vibration, which further proved the existence and reaction between $\mathrm{Ag}$ and long-chain alkyl of PMMA. In addition, shifts in $\mathrm{C}=\mathrm{O}$ ester carbonyl group, $\mathrm{C}=\mathrm{C}, \mathrm{CH}_{3}$, and $\mathrm{C}-\mathrm{C}$ for the AgNP/PMMA nanocomposite thin films were also seen, which indicated the modification of PMMA thin film structures by the AgNPs [23, 27-29].

3.5. The Thermal Gravimetric Analysis. TGA estimations were carried out on Ag/PMMA nanocomposite and pure PMMA. The samples of settled weight were heated at the rate $100^{\circ} \mathrm{C} / \mathrm{min}$ from room temperature to $800^{\circ} \mathrm{C}$, which was in between the boiling point of the solvent and degradation temperature of the polymer. Figure 4 demonstrates two particular phases of the watched weight and underlying weight reduction was figured to be around $20 \%$ and $5 \%$ for PMMA and Ag/PMMA, respectively, from room temperature to $300^{\circ} \mathrm{C}$.

The weight reduction up to this temperature was credited to low subatomic weight oligomers, loss of moisture, 


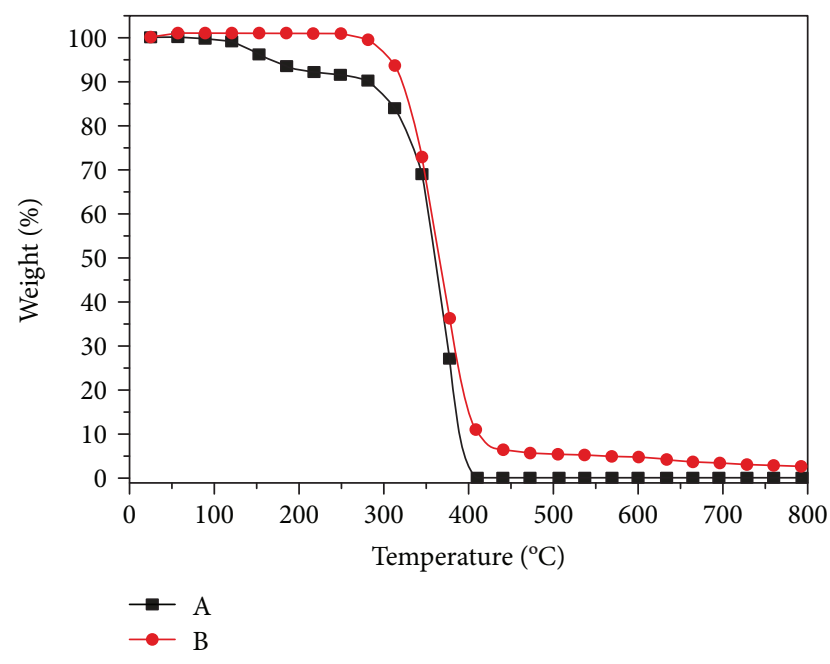

Figure 5: (a) TGA thermogram of polymer PMMA. (b) TGA thermogram of greener AgNP/PMMA nanocomposite.

and leftover solvent. The second weight reduction demonstrated the degradation of pure PMMA (Figure 5(a)) at over $360^{\circ} \mathrm{C}$, which completely disintegrated at $400^{\circ} \mathrm{C}$, while the Ag/PMMA nanocomposite disintegrated at over $800^{\circ} \mathrm{C}$ (Figure 5(b)). The second significant weight reduction was ascribed to the basic deterioration of the polymer. The weight reductions of Ag/PMMA nanoparticles and pure PMMA are shown in Figures 5(a) and 5(b). The thermogravimetric investigation of the Ag/PMMA nanocomposite indicated a decomposition profile beginning at $400^{\circ} \mathrm{C}$ and proceeding until over $800^{\circ} \mathrm{C}$. This demonstrated that the high thermal stability of the polymer was enhanced by the presence of $\mathrm{Ag}$ as a nanofiller [30].

3.6. The Microbial Growth Results. In this work, microbiological testing was performed for tap water and treated water with a nanocomposite film utilizing different types of media. The outcomes demonstrated that plates with water treated with the nanocomposite film had no growth of microorganisms unlike plates with ordinary tap water (Figure 6). This was because the greener AgNPs in the film in treated water had particularly natural substances like nitrogen and phosphorus, which were fundamental to the response of bacterial metabolism.

The greener nanocomposite showed phenomenal microorganism activity contingent on the nanoparticle size as this parameter changed the surface area in contact with the bacterial species. The presence of green AgNPs in the nanocomposite clarifies the antimicrobial property found in the synthesized nanocomposite film.

The greener nanocomposite demonstrated antibacterial activity depending on nanoparticle size as this parameter changed the surface area in contact with the bacterial species. The presence of green AgNPs in the nanocomposite explained the antimicrobial properties found in the prepared nanocomposite.

Morones et al. [31] announced that small sized nanoparticles went through cell membranes, leading to cell malfunction. It can be reasoned that the synthesized greener

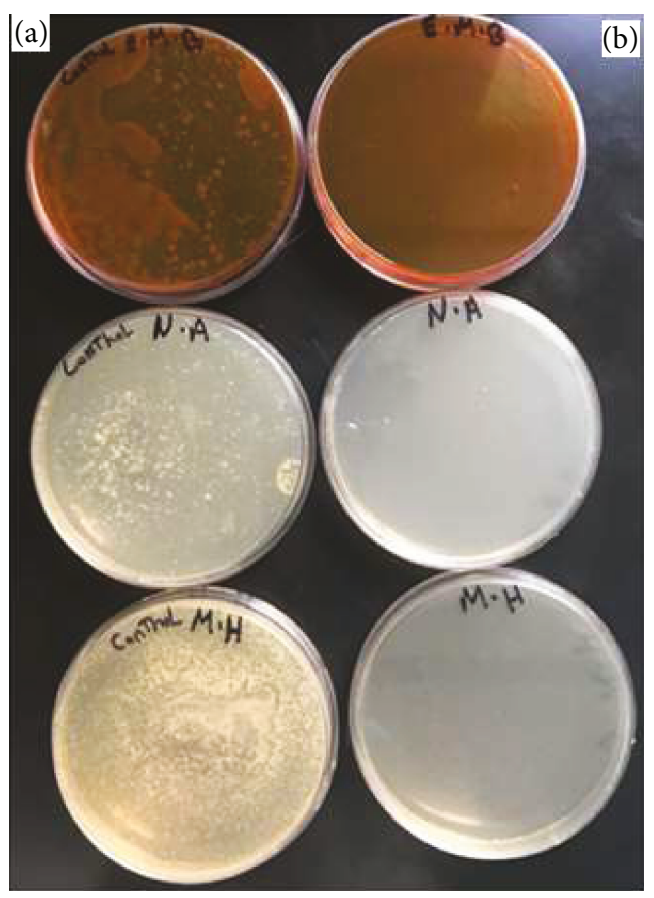

FIGURE 6: The results of microbial growth for both (a) untreated tap water and (b) water treated with a nanocomposite film utilizing eosin methylene blue (E.M.B) agar, nutrient agar (N.A), and Muller-Hinton (M.H) agar media.

AgNP/PMM nanocomposite was effective against microbes, considering that nanoparticles were uniformly dispersed in the polymer matrix. Generally, the system of inhibitory impacts of $\mathrm{Ag}$ particles on microorganisms is incompletely known. A few investigations have detailed that the positive charge on the Ag particle is pivotal for its antimicrobial activity through electrostatic attraction between the negative charge on the cell membrane of the microorganism and positively charged nanoparticles. The antibacterial mechanism of AgNPs is not clearly defined. There are several theories presented in many literatures. One of these theories is AgNPs can cause damage and leakage of intracellular material by attaching to the cell membrane. The second theory mentioned that the cause of cell death is the formation of free radicals by AgNPs since they have the ability to generate pores in the membrane. The third theory considers that the damages of the integrity and permeability of the membrane are caused by the release of silver ions from nanoparticles and that these ions can react with functional protein molecules and DNA; hence, they interfere with DNA metabolism and replication resulting in cell death [32].

Strong antibacterial activity against bacteria has been revealed by nanocomposite film as has been reported in several studies [33] Also, the AgNP nanocomposite antibacterial effects have been reported in numerous studies with different theories; the most probable scenario seems to be that silver ions bind to the bacterial cell membrane and damage it by interfering with membrane receptors and with bacterial electron transport. Another scenario is that the killing effect of the bacteria is due to contact killing [34, 35]. Consequently, this effortless approach for producing greener 
AgNP/polymer nanocomposite film is valuable in many industrial applications. The technique permits the utilization of a nontoxic, cheap, ecofriendly, bioavailable material [26].

\section{Conclusions}

The introduced work demonstrated the fast greener synthesis of AgNPs utilizing Trigonella foenum-graecum seeds and their composite with the PMMA polymer. The technique here is nontoxic, ecologically cordial, and straightforward and involves minimal effort and has no lethal chemicals. The formation of greener AgNPs was determined by TEM and UV-Vis spectroscopy, where surface plasmon absorption maxima can be seen at $339 \mathrm{~nm}$ in the UV-Vis range. Zetasizer demonstrated the average size of the resulting nanoparticles to be $83.01 \mathrm{~nm}$. The nanocomposite was characterized utilizing FTIR spectroscopy and XRD techniques. TGA was used to explore the thermal stability and interfacial connection between AgNPs and the polymer matrix. TGA demonstrated that AgNP/PMMA nanocomposite has higher thermal stability than that of the PMMA polymer. The nanocomposite films showed significant antibacterial activity bacteria that exhibited no growth of microbes in treated water. This guarantees a promising potential utilization of the nanocomposite in water decontamination, channels, and water stockpiling, in addition to water and wastewater treatment.

\section{Data Availability}

All our data used to support the findings of this study are included within the article.

\section{Conflicts of Interest}

The authors declare that there are no conflict of interest regarding the publication of this paper.

\section{Acknowledgments}

The authors are thankful to the financial and logistic support of the King Abdullah Institute for Nanotechnology and the Deanship of Scientific Research, King Saud University, Riyadh, Saudi Arabia.

\section{References}

[1] T. J. Brunner, P. Wick, P. Manser et al., "In vitro cytotoxicity of oxide nanoparticles: comparison to asbestos, silica, and the effect of particle solubility," Environmental Science \& Technology, vol. 40, no. 14, pp. 4374-4381, 2006.

[2] P. L. Kashyap, X. Xiang, and P. Heiden, "Chitosan nanoparticle based delivery systems for sustainable agriculture," International Journal of Biological Macromolecules, vol. 77, pp. 36-51, 2015.

[3] R. C. Monica and R. Cremonini, "Nanoparticles and higher plants," Caryologia, vol. 62, no. 2, pp. 161-165, 2009.

[4] Y. Ju-Nam and J. R. Lead, "Manufactured nanoparticles: an overview of their chemistry, interactions and potential environmental implications," Science of The Total Environment, vol. 400, no. 1-3, pp. 396-414, 2008.

[5] Siddartha, Azim, Rahul, and Shyam, "Silver nanoparticles for a new generation of antimicrobial drug delivery," International Journal Advance Research in Science and Engineering, vol. 3, no. 4,2014

[6] N. Singh and P. K. Khanna, Materials Chemistry and Physics, vol. 104, no. 2-3, pp. 367-372, 2007.

[7] V. V. Vodnik, J. V. Vuković, and J. M. Nedeljković, “Synthesis and characterization of silver-poly(methylmethacrylate) nanocomposites," Colloid and Polymer Science, vol. 287, no. 7, pp. 847-851, 2009.

[8] N. D. Singho, N. A. C. Lah, M. R. Johan, and R. Ahmad, "Enhancement of the refractive index of silver nanoparticles in poly (methyl methacrylate)," International Journal of Research in Engineering and Technology, vol. 1, no. 4, 2012.

[9] I. Armentano, M. Dottori, E. Fortunati, S. Mattioli, and J. M. Kenny, "Biodegradable polymer matrix nanocomposites for tissue engineering: a review," Polymer Degradation and Stability, vol. 95, no. 11, pp. 2126-2146, 2010.

[10] D. R. Paul and L. M. Robeson, "Polymer nanotechnology: nanocomposites," Polymer, vol. 49, no. 15, pp. 3187-3204, 2008.

[11] X. Shi, B. Sitharaman, Q. P. Pham et al., "Fabrication of porous ultra-short single-walled carbon nanotube nanocomposite scaffolds for bone tissue engineering," Biomaterials, vol. 28, no. 28, pp. 4078-4090, 2007.

[12] P. Basak, P. Das, S. Biswas, N. C. Biswas, and G. K. D. Mahapatra, "Green synthesis and characterization of gelatin-PVA silver nanocomposite films for improved antimicrobial activity," IOP Conference Series: Materials Science and Engineering, vol. 410, article 012019, 2018.

[13] M. R. Vengatesan and V. Mittal, "Nanoparticle- and nanofiber-based polymer nanocomposites: an overview," in Spherical and Fibrous Filler Composites, V. Mittal, Ed., Wiley-VCH, First Edition edition, 2016.

[14] P. Singh, Y.-J. Kim, D. Zhang, and D.-C. Yang, "Biological synthesis of nanoparticles from plants and microorganisms," Trends in Biotechnology, vol. 34, no. 7, pp. 588-599, 2016.

[15] X.-F. Zhang, Z.-G. Liu, W. Shen, and S. Gurunathan, "Silver nanoparticles: synthesis, characterization, properties, applications, and therapeutic approaches," International Journal of Molecular Sciences, vol. 17, no. 9, p. 1534, 2016.

[16] D. A. H. Hanaor and C. C. Sorrell, "Sand supported mixed-phase $\mathrm{TiO}_{2}$ photocatalysts for water decontamination applications," Advanced Engineering Materials, vol. 16, no. 2, pp. 248-254, 2014.

[17] D. Hanaor, M. Michelazzi, J. Chenu, C. Leonelli, and C. C. Sorrell, "The effects of firing conditions on the properties of electrophoretically deposited titanium dioxide films on graphite substrates," Journal of the European Ceramic Society, vol. 31, no. 15, pp. 2877-2885, 2011.

[18] M. R. Bindhu and M. Umadevi, "Synthesis of monodispersed silver nanoparticles using Hibiscus cannabinus leaf extract and its antimicrobial activity," Spectrochimica Acta Part A: Molecular and Biomolecular Spectroscopy, vol. 101, pp. 184190, 2013.

[19] A. Ahmad, P. Mukherjee, S. Senapati et al., "Extracellular biosynthesis of silver nanoparticles using the fungus Fusarium oxysporum," Colloids and Surfaces, B: Biointerfaces, vol. 28, no. 4, pp. 313-318, 2003. 
[20] S. Goyal, N. Gupta, A. Kumar, S. Chatterjee, and S. Nimesh, "Antibacterial, anticancer and antioxidant potential of silver nanoparticles engineered using Trigonella foenum-graecum seed extract," IET Nanobiotechnology, vol. 12, no. 4, pp. 526533, 2018.

[21] N. D. Singho, M. R. Johan, and N. A. C. Lah, "Temperaturedependent properties of silver-poly(methylmethacrylate) nanocomposites synthesized by in-situ technique," Nanoscale Research Letters, vol. 9, no. 1, p. 42, 2014.

[22] N. Faraji, W. M. M. Yunus, A. Kharazmi, and E. Saion, "Third-order nonlinear optical properties of silver nanoparticles mediated by chitosan," Optik, vol. 125, no. 12, pp. 2809-2812, 2014.

[23] A. Kadhim, H. R. Humud, and L. Abd al Kareem, "XRD and FTIR studies for Ag/PMMA nano composite thin films," International Journal of Computation and Applied Sciences, vol. 1, no. 2, pp. 21-27, 2016.

[24] E. Alsharaeh, "Polystyrene-poly(methyl methacrylate) silver nanocomposites: significant modification of the thermal and electrical properties by microwave irradiation," Materials, vol. 9, no. 6, p. 458, 2016.

[25] R. Shah, A. Kausar, and B. Muhammad, "Exploration of polythiophene/graphene, poly(methyl methacrylate)/graphene and polythiophene-co- poly(methyl methacrylate)/graphene nanocomposite obtained via in-situ technique," Journal of Plastic Film \& Sheeting, vol. 31, no. 2, pp. 144157, 2015.

[26] M. A. Awad, W. K. Mekhamer, N. M. Merghani et al., "Green synthesis, characterization, and antibacterial activity of silver/polystyrene nanocomposite," Journal of Nanomaterials, vol. 2015, Article ID 943821, 6 pages, 2015.

[27] Y. Li, L. Gao, W. Chen et al., "Preparation and characterization of Ag2S/PMMA nanocomposites by microemulsion," Acta Metallurgica Sinica (English Letters), vol. 27, no. 1, pp. 175179, 2014.

[28] T. P. Kasih, Development of Novel Potential of Plasma Polymerization Techniques for Surface Modification, Gunma University, Ph.D Thesis, 2007.

[29] M. Haris, S. Kathiresan, and S. Mohan, "FT-IR and FT-Raman spectra and normal coordinate analysis of poly methyl methacrylate," Der Pharma Chemica, vol. 2, pp. 316-323, 2010.

[30] Z. H. Mbhele, M. G. Salemane, C. G. C. E. van Sittert, J. M. Nedeljković, V. Djoković, and A. S. Luyt, "Fabrication and characterization of silver-polyvinyl alcohol nanocomposites," Chemistry of Materials, vol. 15, no. 26, pp. 50195024, 2003.

[31] J. R. Morones, J. L. Elechiguerra, A. Camacho et al., "The bactericidal effect of silver nanoparticles," Nanotechnology, vol. 16, no. 10, pp. 2346-2353, 2005.

[32] S. Prabhu and E. K. Poulose, "Silver nanoparticles: mechanism of antimicrobial action, synthesis, medical applications, and toxicity effects," International Nano Letters, vol. 2, no. 1, p. 32, 2012.

[33] I. Armentano, C. R. Arciola, E. Fortunati et al., "The interaction of bacteria with engineered nanostructured polymeric materials: a review," The Scientific World Journal, vol. 2014, Article ID 410423, 18 pages, 2014.
[34] B. Jia, Y. Mei, L. Cheng, J. Zhou, and L. Zhang, "Preparation of copper nanoparticles coated cellulose films with antibacterial properties through one-step reduction," ACS Applied Materials and Interfaces, vol. 4, no. 6, pp. 28972902, 2012.

[35] E. Fortunati, F. D’Angelo, S. Martino, A. Orlacchio, J. M. Kenny, and I. Armentano, "Carbon nanotubes and silver nanoparticles for multifunctional conductive biopolymer composites," Carbon, vol. 49, no. 7, pp. 2370-2379, 2011. 


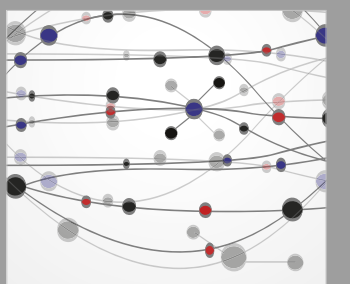

The Scientific World Journal
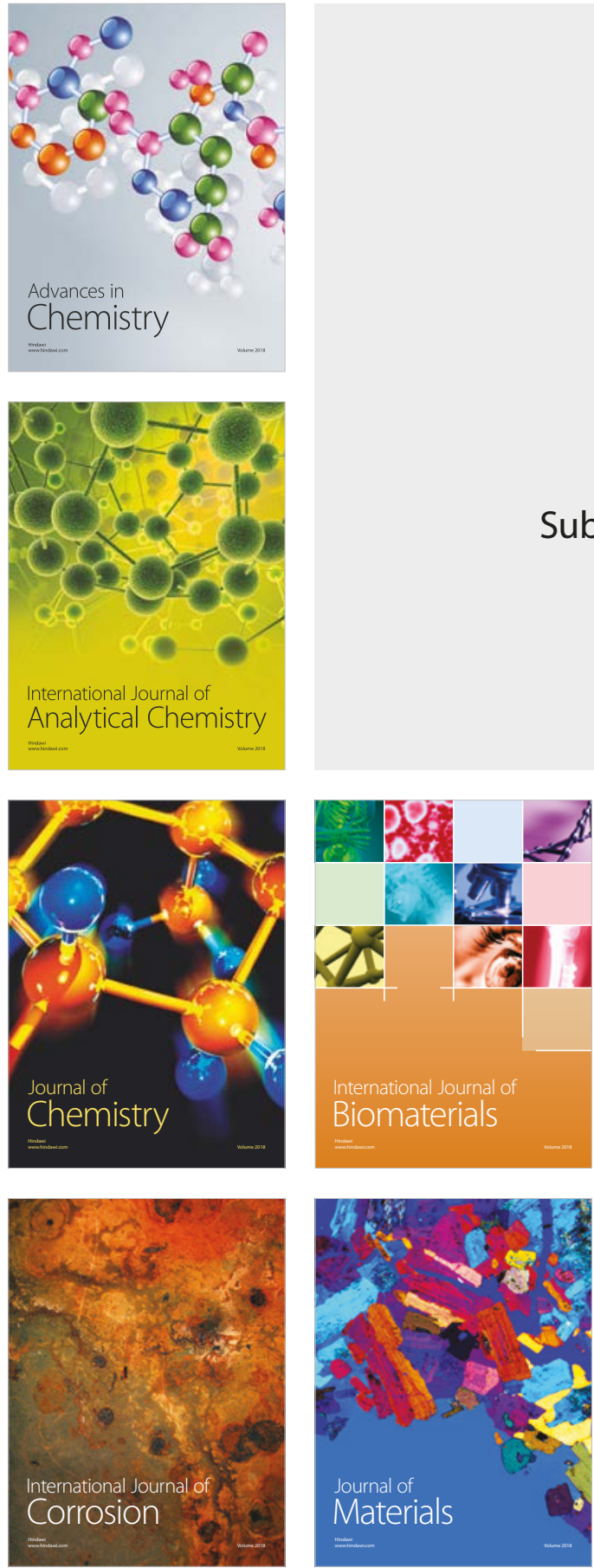

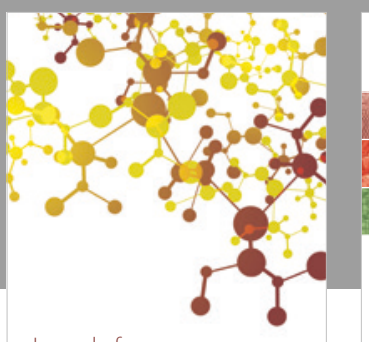

Journal of

Applied Chemistry
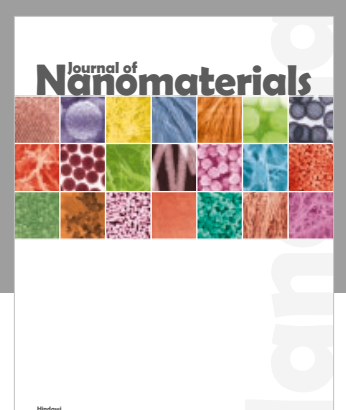

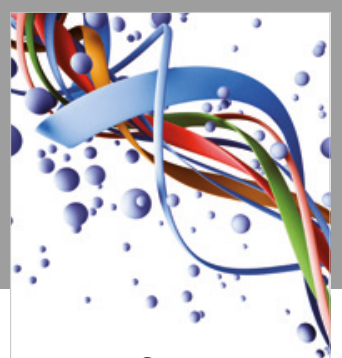

Scientifica

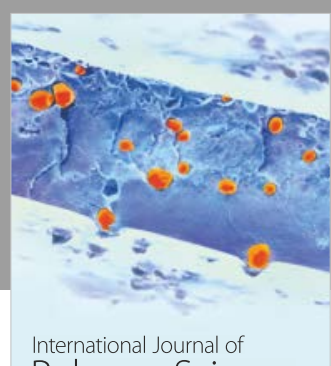

Polymer Science

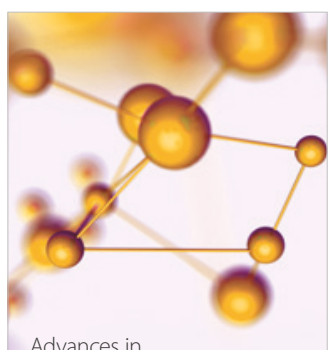

Physical Chemistry
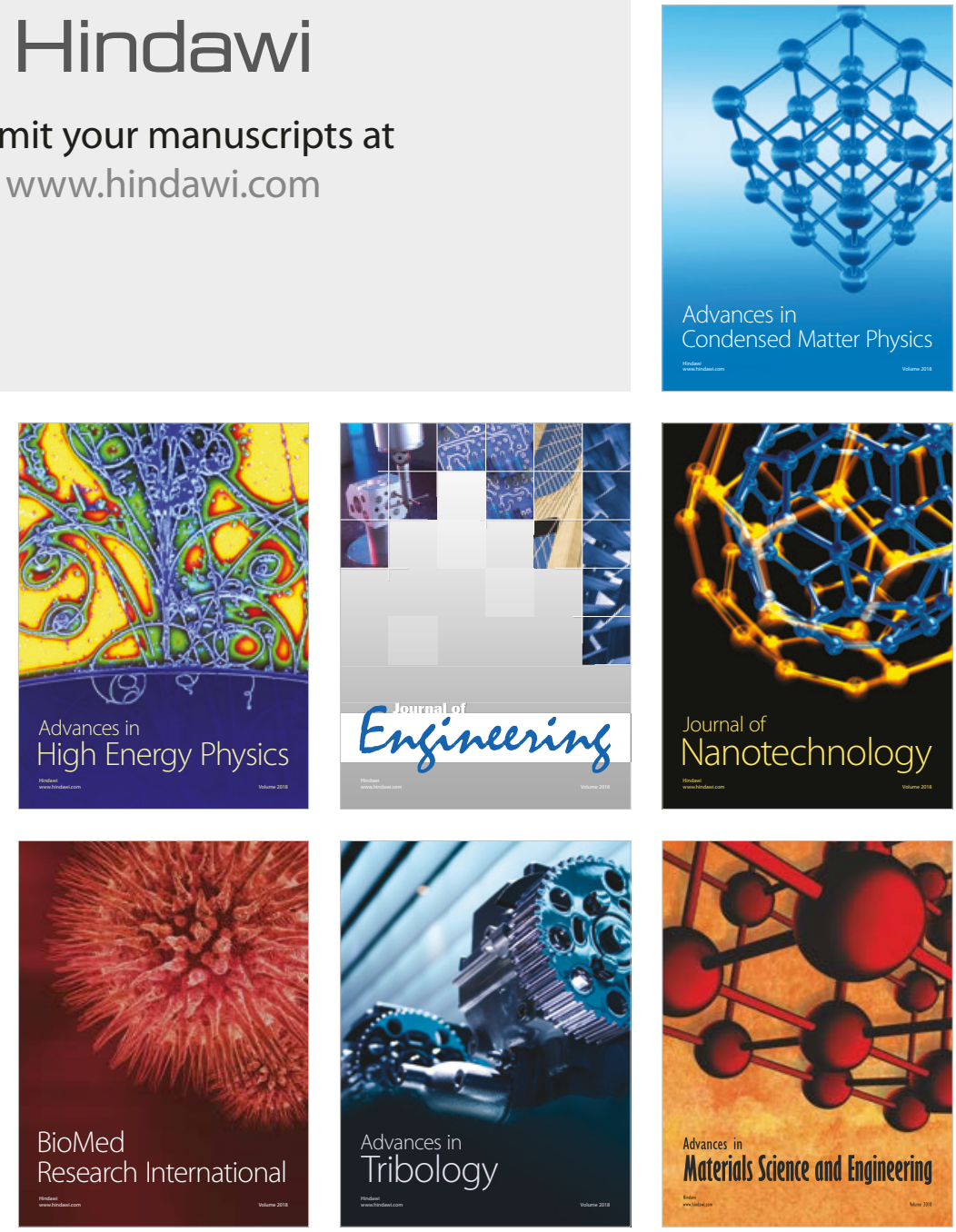